\title{
Observations of concentrated generator regions in the nightside magnetosphere by Cluster/FAST conjunctions
}

\author{
M. Hamrin ${ }^{1}$, O. Marghitu ${ }^{2,3}$, K. Rönnmark ${ }^{1}$, B. Klecker ${ }^{3}$, M. André ${ }^{4}$, S. Buchert ${ }^{4}$, L. M. Kistler ${ }^{5}$, J. McFadden ${ }^{6}$, \\ H. Rème ${ }^{7}$, and A. Vaivads ${ }^{4}$ \\ ${ }^{1}$ Department of Physics, Umeå University, Umeå, Sweden \\ ${ }^{2}$ Institute for Space Sciences, Bucharest, Romania \\ ${ }^{3}$ Max-Planck-Institut für extraterrestrische Physik, Garching, Germany \\ ${ }^{4}$ Swedish Institute of Space Physics, Uppsala, Sweden \\ ${ }^{5}$ Space Science Center, University of New Hampshire, NH, Durham, USA \\ ${ }^{6}$ Space Sciences Lab., University of California at Berkley, USA \\ ${ }^{7}$ CESR-CNRS, Toulouse, France
}

Received: 19 April 2005 - Revised: 21 November 2005 - Accepted: 10 January 2006 - Published: 23 March 2006

\begin{abstract}
Here and in the companion paper, Marghitu et al. (2006), we investigate plausible auroral generator regions in the nightside auroral magnetosphere. In this article we use magnetically conjugate data from the Cluster and the FAST satellites during a 3.5-h long event from 19-20 September 2001. Cluster is in the Southern Hemisphere close to apogee, where it probes the plasma sheet and lobe at an altitude of about $18 R_{E}$. FAST is below the acceleration region at approximately $0.6 R_{E}$. Searching for clear signatures of negative power densities, $\boldsymbol{E} \cdot \boldsymbol{J}<0$, in the Cluster data we can identify three concentrated generator regions (CGRs) during our event. From the magnetically conjugate FAST data we see that the observed generator regions in the Cluster data correlate with auroral precipitation. The downward Poynting flux observed by Cluster, as well as the scale size of the CGRs, are consistent with the electron energy flux and the size of the inverted-V regions observed by FAST. To our knowledge, these are the first in-situ observations of the crossing of an auroral generator region. The main contribution to $\boldsymbol{E} \cdot \boldsymbol{J}<0$ comes from the GSE $E_{y} J_{y}$. The electric field $E_{y}$ is weakly negative during most of our entire event and we conclude that the CGRs occur when the duskward current $J_{y}$ grows large and positive. We find that our observations are consistent with a local southward expansion of the plasma sheet and/or rather complicated, 3-D wavy structures propagating over the Cluster satellites. We find that the plasma is working against the magnetic field, and that kinetic energy is being converted into electromagnetic energy. Some of the energy is transported away as Poynting flux.
\end{abstract}

Correspondence to: M. Hamrin

(hamrin@space.umu.se)
Keywords. Magnetospheric physics (Auroral phenomena; Magnetosphere-ionosphere interactions; Magnetotail boundary layers)

\section{Introduction}

During the expansion phase of a substorm, large amounts of plasma are convected towards the Earth as the geomagnetic field returns to a more dipolar configuration. Bursty bulk flows with speeds larger than $400 \mathrm{~km} / \mathrm{s}$ have been observed in the central plasma sheet (Angelopoulos et al., 1992). As the flow reaches the inner boundary of the plasma sheet, the flow is diverted and slowed down, and the plasma starts to flow towards local dawn and dusk. This can result in strongly sheared plasma flows and sharp density gradients. In generator regions, the free energy associated with such plasma flows and pressure gradients can be converted into electromagnetic energy, which is transported from the generator region as a Poynting flux toward the two main load regions where energy is dissipated. These load regions are characterized by a positive value of the scalar product between the electric field and the current density, $\boldsymbol{E} \cdot \boldsymbol{J}$, and they can be summarized as: 1) horizontal currents in the upper atmosphere/ionosphere, and 2) the acceleration region where parallel electric fields, $E_{\|}$, accelerate electrons along the magnetic field. Some of the Poynting flux can also be reflected back toward the generator region in the magnetosphere.

A standard picture of the auroral current circuit is schematically sketched in Fig. 1, indicating the magnetosphereionosphere coupling. The circuit is powered by a generator region characterized by a negative value of $\boldsymbol{E} \cdot \boldsymbol{J}$. Early

Published by Copernicus GmbH on behalf of the European Geosciences Union. 


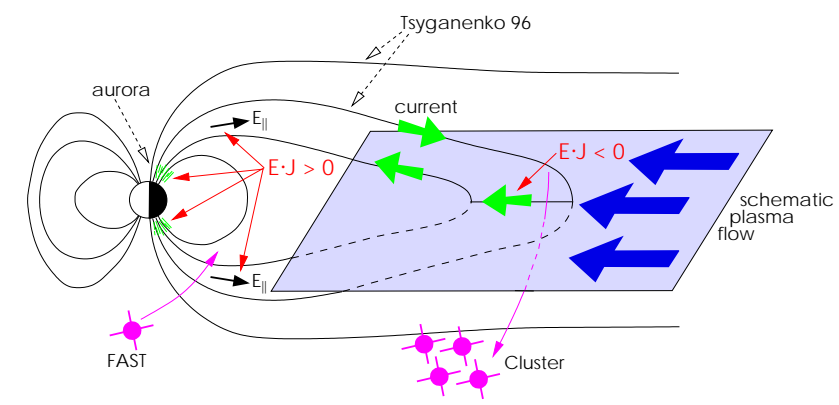

Fig. 1. A schematic sketch of the auroral current circuit. The generator region $(\boldsymbol{E} \cdot \boldsymbol{J}<0)$ in the magnetosphere powers the loads $(\boldsymbol{E} \cdot \boldsymbol{J}>0)$ in the acceleration region and the auroral ionosphere. In this article we analyze conjugate data from the Cluster fleet and the FAST spacecraft when Cluster is in the magnetosphere at an altitude of about 18 Earth radii and FAST passes below the auroral acceleration region at an altitude of about 0.6 Earth radii. Both Cluster and FAST are in the Southern Hemisphere during our event (22:00 UT, on 19 September to 01:30, on 20 September 2001).

studies of the generator region and its coupling to the ionosphere were done by Boström (1975) and Rostoker and Boström (1976), who discussed the basic concept of an MHD generator. Since the 70's, further investigations of the generator region have been conducted. The development within this area has been reviewed (e.g. Lysak, 1990; Vogt, 2002; Paschmann et al., 2003).

An interesting question concerns whether the generator is confined to the equatorial region or extends to non-equatorial latitudes. Large-scale MHD simulations (Birn et al., 1999) suggest that although strong plasma flows mainly exist in the equatorial plane, the magnetic shear or twist is generated predominantly outside of this flow region, above and below the equatorial plane. Since these magnetic disturbances signify field-aligned currents, it seems plausible that generator signatures can be found outside the equatorial plane. Several regions has been suggested as possible hosts for the generator in the middle or outer magnetosphere, for example, the low-latitude boundary layer, the plasma sheet and the plasma sheet boundary layer. The generator have also been suggested to exist closely above the acceleration region (Janhunen, 2000). Borovsky (1993) discussed 10 different generator mechanisms and their location in the Earth's magnetosphere.

Attempts have been made to determine the location of the generator by field line mapping between the auroral ionosphere and the outer magnetosphere (see, e.g. Lu et al., 2000). Such a method is of course limited by the accuracy of the magnetic field model, but a more fundamental problem is that it assumes the currents and the associated Poynting flux to be strictly field-aligned. However, mechanical forces, such as shear flows and pressure gradients, can cause the current to flow significant distances perpendicular to the unperturbed field lines. Hence, the identification of possible generator regions in the Earth's magnetosphere only by field line mapping from the auroral ionosphere to a magnetospheric source is not sufficient. There is obviously a need for in-situ observations that can identify generator regions in the magnetosphere.

A moderately strong aurora corresponds to an electron energy flux of about $10 \mathrm{~mW} / \mathrm{m}^{2}$ in the auroral ionosphere. According to the discussion in Marghitu et al. (2006), this corresponds to a power density in the generator region of $10^{-13} \mathrm{~W} / \mathrm{m}^{3} \lesssim|\boldsymbol{E} \cdot \boldsymbol{J}| \lesssim 10^{-12} \mathrm{~W} / \mathrm{m}^{3}$. For typical values of the electric field and the current density near the tail midplane, $1 \mathrm{mV} / \mathrm{m}$ and $1 \mathrm{nA} / \mathrm{m}^{2}$, this is close to the limits of accuracy of the Cluster instruments. Accurate estimates of the power density are hence difficult to obtain, but the sign and the general trends of $\boldsymbol{E} \cdot \boldsymbol{J}$ should be easier to estimate.

In this article we investigate possible generator regions in the magnetosphere by direct observations. The Cluster mission is in many respects suitable for studies of the generator region. For example, the full current density vector $\boldsymbol{J}=\nabla \times \boldsymbol{B} / \mu_{0}$ can be derived from the simultaneous magnetic field measurements from the four spacecraft. According to basic electrodynamics, the sign of $\boldsymbol{E} \cdot \boldsymbol{J}$ can be used to distinguish between loads and generators, and we identify possible generator regions by searching for signatures of $\boldsymbol{E} \cdot \boldsymbol{J}<0$ in the data. In this article we present data from the Cluster satellites for a 3.5-h long event during 19-20 September 2001, when the satellites are in the Southern Hemisphere probing the plasma sheet and the lobe. To verify that the Cluster data correlates with auroral precipitation, we study magnetic conjugate, low-altitude electron data from the FAST satellite. It should be noted that accelerated auroral electrons and the high altitude magnetospheric generator need not be located on exactly the same field line. The location of the aurora with respect to the generator, depends on, for example, how and where the current circuit is closed. However, such a discussion is outside the scope of this article.

An additional generator region is discussed in detail in our companion paper, Marghitu et al. (2006), referred throughout in this article as M06.

\section{Data processing/methods}

In this article we investigate magnetically conjugate data (from 22:00 UT, 19 September, to 01:30 UT, 20 September 2001) from the Cluster fleet and the FAST satellite in the nightside auroral region in the Southern Hemisphere. The spacecraft instrumentation and data processing/methods used are briefly summarized in this section. However, a more detailed discussion of these items can be found in M06.

Cluster crosses auroral field lines at $\sim 23: 00$ MLT, well above the acceleration region at an altitude of about $18 R_{E}$ and FAST is below the acceleration region at approximately $0.6 R_{E}$. Due to FAST's short orbiting period $(\sim 130 \mathrm{~min})$ and the favourable orientation of the orbital planes of the 
satellites, there are two close magnetic field conjunctions between FAST and Cluster during our event. The conjunctions between the satellites occur at 22:23 UT (19 September 2001) and 00:29 UT (20 September 2001).

In Fig. 2 the trajectories of Cluster S/C 1 (green) and the Fast satellite (red), mapped to an altitude of $110 \mathrm{~km}$ are shown. Since the ionospheric footprints of the satellites are located above Antarctica, conjugate ground-based data (magnetic, radar or optical) are not available. Similarly, there are no optical data from the IMAGE or Polar satellites.

We see that the footprints meet practically head on. Cluster moves toward dusk at almost constant latitudes, while FAST moves equatorward toward midnight. For the mapping, the Tsyganenko T96 model has been used with the appropriate conditions for our event. For the first conjunction the ionospheric footprints of Cluster S/C 1 and FAST are approximately at the same latitude, and separated by less than $30 \mathrm{~min}$ in MLT (i.e. $7.5^{\circ}$ or about $250 \mathrm{~km}$ ). When mapped to the Cluster altitude, this difference in azimuth corresponds to $1-2 R_{E}$ (see Fig. 12 in M06). The second conjunction is almost perfect and at 00:29 UT the ionospheric footprints are almost on top of each other.

However, during our event Cluster is at high altitudes and close to apogee. This makes the field line mapping sensitive to magnetic field variations. One should note that the T96 model does not include contributions from the magnetotail motion, such as observed at the Cluster altitude during this event. In Sect. 3 we will see that the Cluster spacecraft is brought in and out of the plasma sheet by the motion of the magnetotail. However, since our event corresponds to a rather low magnetospheric activity $\left(K_{p}=1\right)$, we believe that the magnetic field variations are small enough to make the mapping satisfactory. Moreover, in Sect. 3 we will see that similar signatures in the particle data from both Cluster and FAST can also be used to support the mapping. Hence, we believe that the ionospheric footprints of FAST and Cluster are very close in the ionosphere for both conjunctions, even though they need not be located on exactly the same field line. On the other hand, as stated in the Introduction, we cannot assume that the generator and the accelerated auroral electrons are located on the same field line. Therefore, an exact conjunction between the satellites is not needed.

As shown by Slavin et al. (2003) a small substorm started at 20:39 UT on 19 September 2001, with the recovery phase initiated at 22:15 UT. It should be noted that the magnetospheric activity is low during the entire 3.5 -h long event, with a $K_{p}$ index of about 1 . This suggests that it is reasonable to assume that the position of the generator region and the auroral flux-tubes are rather stationary, at least during the time it takes for the electrons to move between Cluster and FAST ( $\sim 10-20$ s). Hence, we can infer that Cluster and FAST observe almost the same auroral flux-tubes, although the distance along the magnetic field line between the satellites is large.

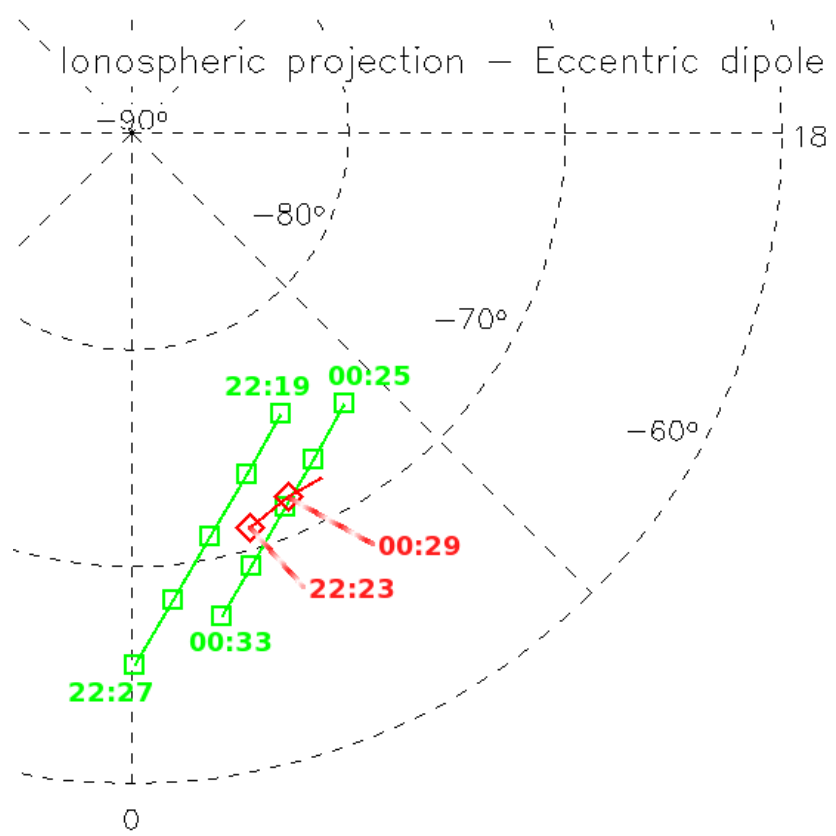

Fig. 2. Trajectories mapped to the ionosphere ( $110 \mathrm{~km}$ altitude) of Cluster S/C 1 (red) and the FAST satellite (green) for the two conjunctions at 22:23 UT (19 September 2001) and 00:29 UT (20 September 2001). Every $2 \mathrm{~min}$ a green square is plotted to indicate the FAST location. The T96 magnetic field model is used for the mapping.

\subsection{Cluster data}

The Cluster mission (Escoubet et al., 1997, 2001) is in many respects suitable for studies of the generator region. For example, using the curlometer method the full current density vector $\boldsymbol{J}=\nabla \times \boldsymbol{B} / \mu_{0}$ can be derived from the simultaneous magnetic field measurements on the four spacecraft (Balogh et al., 2001). As a quality indicator for the estimated current, the quantity $|\nabla \cdot \boldsymbol{B}| /|\nabla \times \boldsymbol{B}|$ can be used. In theory, this quantity should be identically zero, but in practice it can vary substantially (Paschmann and Daly, 1998; Dunlop et al., 2002) due to, for example, small-scale variations in the magnetic field and measurement errors. It should be noted that a oneto-one correspondence between the value of $|\nabla \cdot \boldsymbol{B}| /|\nabla \times \boldsymbol{B}|$ and the actual error in the estimated current density is not expected.

The quality of the curlometer current estimate is rather sensitive to the size and shape of the Cluster tetrahedron. Current structures smaller than the size of the tetrahedron cannot be resolved. In our event, the characteristic size of the tetrahedron of $\sim 1500 \mathrm{~km}$ covers a few proton gyroradii (the characteristic proton temperature is $\lesssim 4 \mathrm{keV}$ ). Moreover, during our event the tetrahedron shape is close to equilateral (the planarity and the elongation are never larger than about 0.1 - see Paschmann and Daly (1998) for a more thorough discussion of the planarity and the elongation). Hence, we 
conclude that both the size and the shape of the Cluster tetrahedron are rather optimal during our event.

Note that since the curlometer method cannot resolve spatial variations in the current density smaller than the tetrahedron characteristic size, all Cluster data in this article have been smoothed to remove the rapid fluctuations smaller than $24 \mathrm{~s}$ (roughly the time it takes for the plasma to flow across the Cluster tetrahedron).

Estimates of the power density $\boldsymbol{E} \cdot \boldsymbol{J}$ also depend on the quality of the electric measurements. The electric field, $\boldsymbol{E}$, can be obtained from three different instruments on board Cluster. The Electric Fields and Waves experiment (EFW) (Gustafsson et al., 1997, 2001) and the Electron Drift Instrument (EDI) (Paschmann et al., 2001) are designed to measure the electric field directly. In addition, the electric field can be computed from the drift of low energy plasma ions as detected by the Cluster Ion Spectrometer (CIS) (Rème et al., 2001), on the assumption that the $\boldsymbol{E} \times \boldsymbol{B}$ drift is dominant. The CIS experiment consist of a mass and energy ion spectrometer CODIF (Composition Distribution Function) and a energy ion spectrometer HIA (Hot Ion Analyser). Data from both CODIF and HIA are used in this article.

As discussed in M06 the electric field measurements are particularly difficult in the vicinity of the tail midplane. During our event, the electric field is rather weak and close to the instruments' sensitivity limits of CIS and EFW. Since the magnitude of the magnetic field is too small, the EDI instrument, which measures the drift of a weak test electron beam, does not operate. Moreover, we cannot obtain the full electric field vector from the EFW instrument, since the magnetic field vector is generally too close to the Cluster spin plane that contains the EFW probes.

In this article we use the electric field from both CIS instruments (CODIF and HIA) and from EFW, for analyzing possible generator regions. By comparing the data from these instruments and using the complementarity of the data sets, we can achieve a better characterization of the electric field.

Due to the lack of the full electric field vector from EFW, we have to rely on the CIS/CODIF and HIA instruments for obtaining the power density $\boldsymbol{E} \cdot \boldsymbol{J}$. Since the curlometer current density is expressed as an average value within the Cluster tetrahedron, the electric field should also be averaged over the tetrahedron volume before calculating $\boldsymbol{E} \cdot \boldsymbol{J}$. However, since there is no active CODIF experiment on satellite 2, only S/C 1, 3, and 4 are included in the electric field average. Moreover, the HIA instrument is operating only on S/C 1 and S/C 3. Therefore, the average of the HIA electric field is based on measurements from just these two satellites.

\subsection{FAST data}

In this article we use FAST electron data from the Electron Electrostatic Analyzer (EESA), giving distribution functions covering the complete pitch-angle range (Carlson et al.,
2001). During the event presented in this study, FAST was in Slow Survey mode, implying a time resolution of $2.5 \mathrm{~s}$.

\subsection{Frame of reference and coordinate system}

As discussed in M06 it is appropriate to calculate $\boldsymbol{E} \cdot \boldsymbol{J}$ in the GSE system which is fixed with respect to the Earth, only showing a yearly rotation relative to an inertial system. At least as long as the auroral current circuit is rather stable, i.e., as long as the magnetosphere is not too much disturbed (note that the $K_{p}$ index is low, $K_{p}=1$, during our event), the GSE system should be a suitable choice.

To separate magnetic field-aligned and perpendicular currents, a magnetic field-aligned coordinate system (MAG) is appropriate. The MAG system has one of its axes aligned with the magnetic field at the center of the Cluster tetrahedron, i.e., it is parallel to the magnetic field averaged between all four satellites. To make the MAG system as close as possible to the GSE system, we choose the MAG- $\alpha$ axis to be anti-parallel to the magnetic field and the MAG- $\beta$ axis to be perpendicular both to the magnetic field and the negative of the average Cluster spacecraft speeds. The MAG- $\gamma$ axis completes the right-handed coordinate system.

\section{Observations}

\subsection{Cluster observations}

Between 19 September, 22:00 UT, and 20 September, 01:30 UT, Cluster is close to apogee and crosses auroral field lines well above the acceleration region at an altitude of about $18 R_{E}$. Hence observations from Cluster can be used to probe possible generator regions in the auroral magnetosphere. To see if the generator signatures observed at Cluster are connected to auroral activities in the auroral ionosphere, we examine electron data from the conjugate FAST satellite, which is below the acceleration region at approximately $0.6 R_{E}$.

Overview plots of the Cluster data are presented in Fig. 3. During our 3.5-h long event on 19-20 September 2001, there are two close conjunctions between the FAST satellite and the Cluster fleet. The conjunctions are indicated in Fig. 3 by vertical magenta lines. FAST summary plots for the two conjunctions are given in Fig. 5.

The top panel of Fig. 3 shows the proton energy spectrogram obtained by the CIS/CODIF instrument on S/C 1 . We see that the satellite is in the southern lobe and enters the plasma sheet at about 22:15 UT. This is consistent with the increase in the proton density, and the parallel and the perpendicular proton temperatures, as well as the change in the plasma flow velocity displayed in Figs. 3b and c. A few hours later, around 02:35 UT (not shown in Fig. 3), the spacecraft leave the plasma sheet and return back to the southern lobe again. 
a)

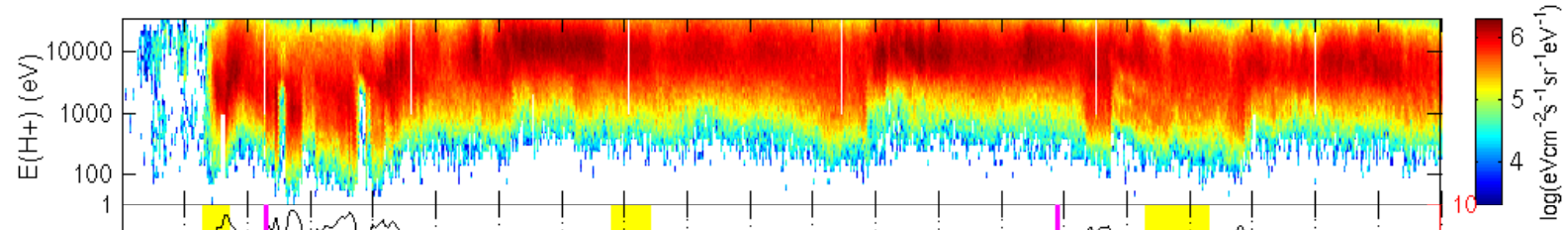

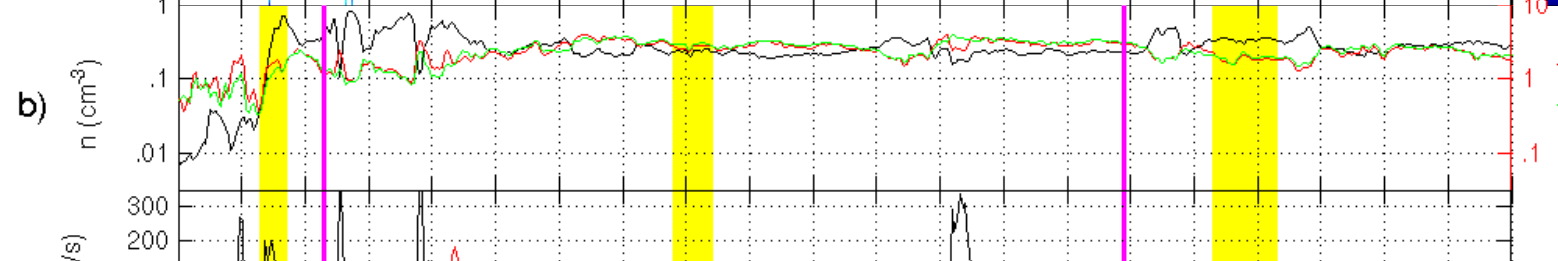

c)

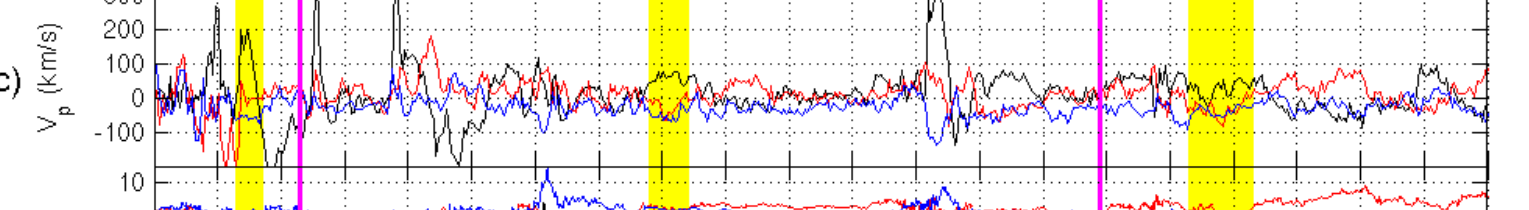

d)

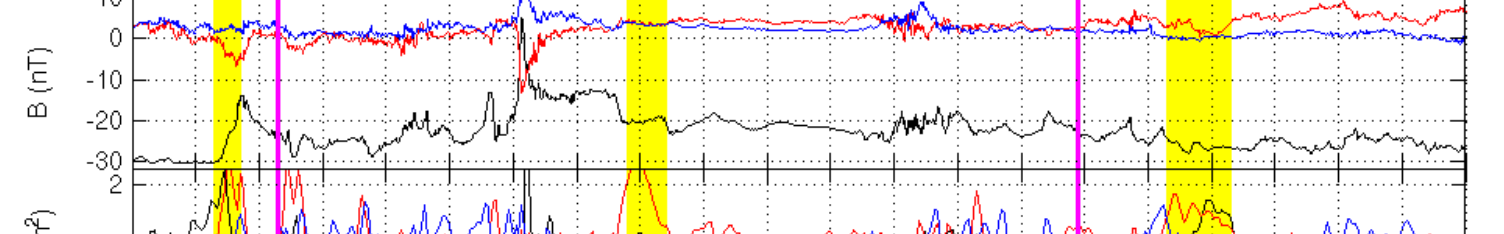

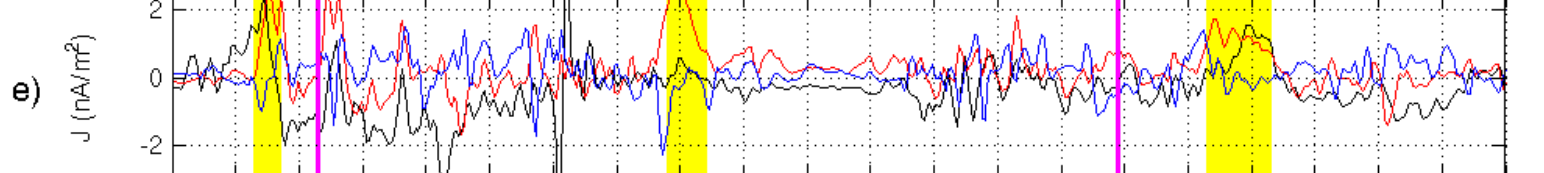

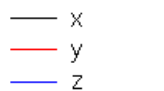

f)

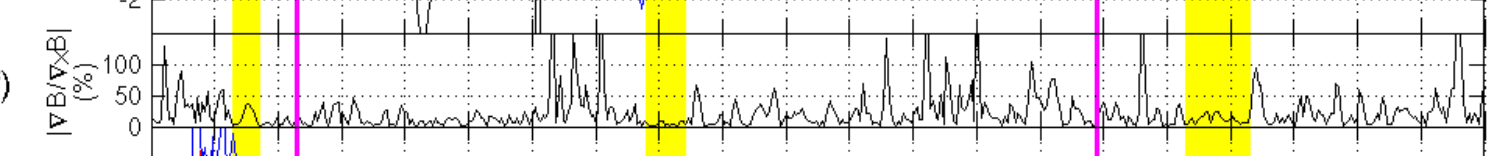

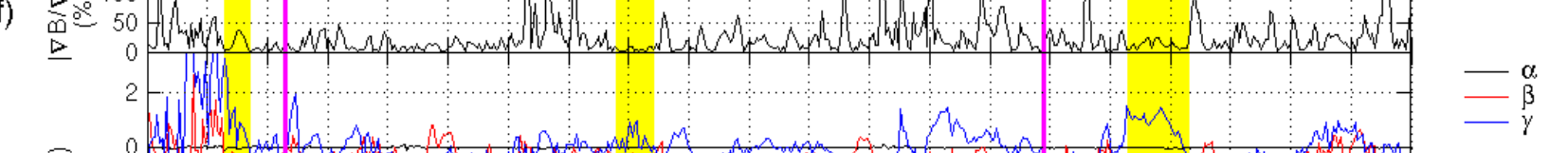
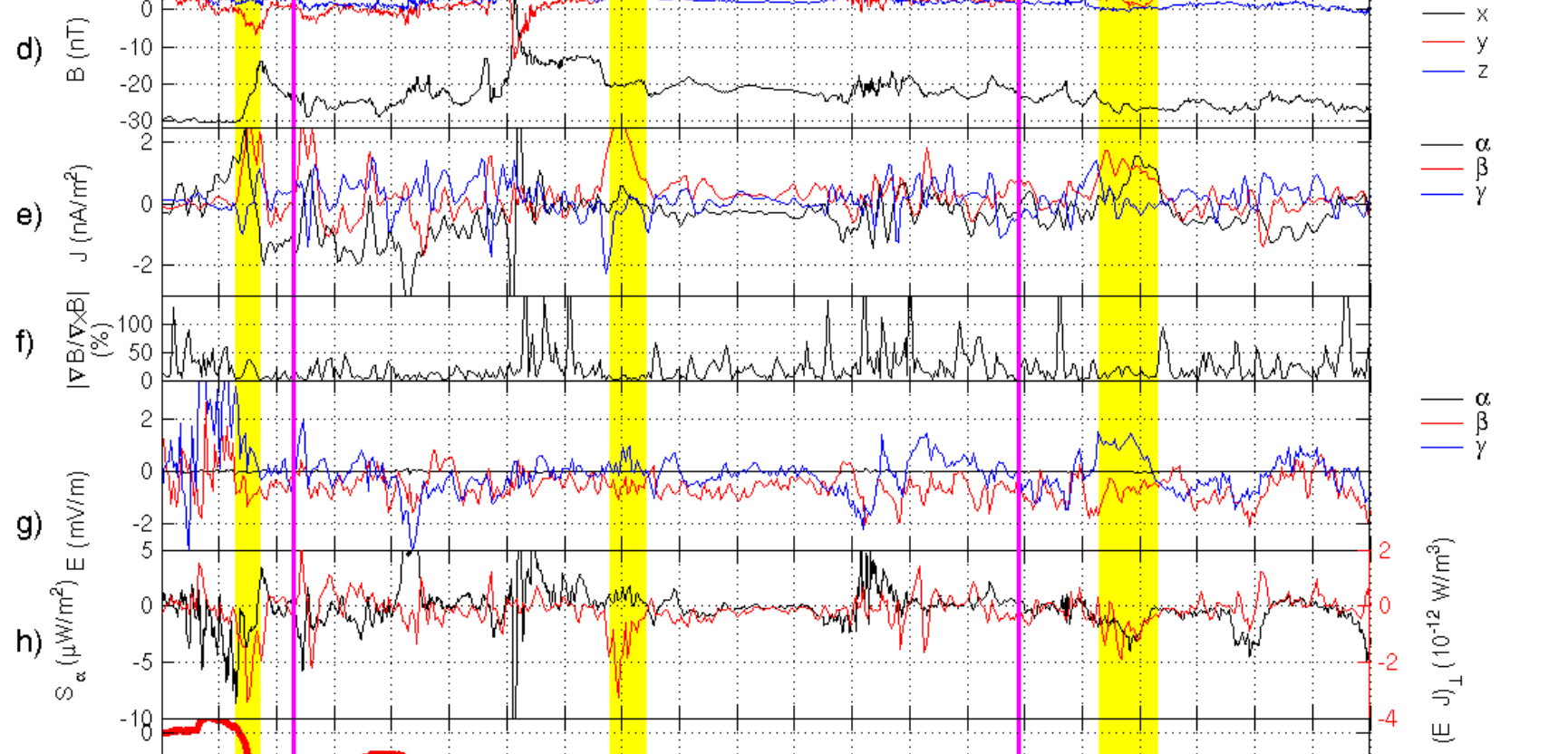

Fig. 3. Cluster data for 19-20 September 2001. All data are smoothed so that variations faster than $24 \mathrm{~s}$ are removed. (a) CODIF proton energy spectrograms from S/C 1; (b) proton density (black), parallel (red) and perpendicular (magenta) proton temperature from CODIF on S/C 1; (c) CODIF plasma flow velocity in GSE from S/C 1; (d) magnetic field in GSE from the FGM experiment on S/C 1; (e) MAG $\alpha$, $\beta$, and $\gamma$ components of the curlometer current density; (f) normalized divergence of the magnetic field; (g) CODIF electric field in MAG average over S/C 1, 3, and 4; (h) field-aligned Poynting flux (black) and power density (red) averaged over S/C 1, 3, and 4 (the CODIF electric field is used); (d) cumulative sum of the power density from the previous panel. The concentrated generator regions CGR1-CGR3 (indicated with yellow bands) appear when there are clear dips in the power density in panel (h) and hence sharp gradients in the cumulative sums in panel (i). The conjunctions with the FAST satellite are shown with the vertical magenta lines. 
The magnetic field from the FGM instrument on Cluster S/C 1 is presented in panel (d) of Fig. 3. Except for the short time period around 23:00 UT, the magnetic field is mainly directed along the negative GSE $x$ direction, i.e. tail-ward. This is consistent with Cluster being in the Southern Hemisphere. Figure $3 e$ contains the current density calculated by the curlometer method from the magnetic field obtained on all four $\mathrm{s} / \mathrm{c}$ and expressed in the magnetic field-aligned coordinate system MAG (see Sect. 2.3 for a definition of the MAG system). For our event the MAG coordinates $(\alpha, \beta, \gamma)$ are closely aligned with the GSE coordinates $(x, y, z)$.

In Fig. $3 \mathrm{f}$ the divergence of the magnetic field, $\nabla \cdot \boldsymbol{B}$, normalized to $\nabla \times \boldsymbol{B}$ is plotted. This quantity can be used as a quality estimate for the curlometer current in the previous panel. During large parts of our 3.5-h long event, we see that $|\nabla \cdot \boldsymbol{B}| /|\nabla \times \boldsymbol{B}|$ is clearly below $50 \%$, although it rises above $100 \%$ at some points. Hence, the estimated current density may not be very accurate at these points. However, for the specific time intervals of interest (indicated with yellow bands in Fig. 3), $|\nabla \cdot \boldsymbol{B}| /|\nabla \times \boldsymbol{B}|$ small.

In panel $(\mathrm{g})$ of Fig. 3 the electric field (averaged over S/C 1, 3, and 4) from the CODIF instrument is shown in MAG coordinates. In the next panel the Poynting flux along the background magnetic field (black) is given, together with the power density, i.e. the scalar product of the average CODIF electric field from panel $(\mathrm{g})$ and the curlometer current density from panel (e). Only contributions from the perpendicular components of the electric field and the current density, $\boldsymbol{E}_{\perp}$ and $\boldsymbol{J}_{\perp}$, are included in the calculation, since the parallel electric field component is presumably negligible. Moreover, the Poynting flux is averaged over S/C 1, 3, and 4.

In Fig. 3h we see three clear regions where the power density drops to negative values. These regions can be identified as Concentrated Generator Regions (CGRs). The CGRs of our event are marked in Fig. 3 with vertical yellow bands. We note that the absolute value of the power density in all of our CGRs is about $1-5 \cdot 10^{-12} \mathrm{~W} / \mathrm{m}^{3}$, which is of the magnitude estimated in Sect. 1.

In Fig. 3i we plot the cumulative sum of $\boldsymbol{E}_{\perp} \cdot \boldsymbol{J}_{\perp}$. It should be noted that we use the cumulative sum only to improve the visibility in the figures. Although Fig. 3h always should be used for detailed studies, it is easier to distinguish the generator regions in Fig. 3i. To confirm the existence of the three CGRs in Figs. 3h and i, we compare the power density obtained from various different estimates of the Cluster electric field. In Fig. 4 we present the cumulative sum of the power density data obtained from all Cluster instruments available for electric field measurements.

The thick red curve in Figs. $3 \mathrm{i}$ and 4 represents the cumulative sum of the power density plotted in Fig. $3 \mathrm{~h}$. Three sharp slopes, corresponding to the three concentrated generator regions, are clearly visible around 22:15, 23:20, and 00:45 UT. During the first three hours of our event, the thick red curve also shows a slowly sloping trend while it is slightly positive during the latter part. However, since the slope of the cumulative sum is rather weak outside the CGRs, the corresponding power density is only slightly different from zero. A more extensive investigation is needed to determine whether this weak slope can be interpreted as an extended generator region, or if it presents just artefacts caused by instrumental errors and random fluctuations. However, this is out of the scope of this article.

Figure 4 gives a more detailed view on the cumulative sum of the power density. The thick red curve is identical with the one plotted in Fig. 3i. The thin solid and dashed red lines correspond to the contribution of $E_{\beta} J_{\beta}$ and $E_{\gamma} J_{\gamma}$, respectively, to the total cumulative sum, $\boldsymbol{E}_{\perp} \cdot \boldsymbol{J}_{\perp}$. From these curves we note that the main contribution to the negative slope in $\boldsymbol{E}_{\perp} \cdot \boldsymbol{J}_{\perp}$ comes from the $\beta$ components of the electric field and the current density.

In addition to the CIS/CODIF instrument there is also the CIS/HIA instrument on S/C 1 and 3. Using the HIA electric field averaged over S/C 1 and 3 we can calculate the corresponding $\boldsymbol{E}_{\perp} \cdot \boldsymbol{J}_{\perp}, E_{\beta} J_{\beta}$ and $E_{z} J_{z}$, shown as the blue lines (thick line, solid line, and dashed line, respectively) in Fig. 4.

There are also electric field measurements from the EFW instrument on board all four Cluster spacecraft. As discussed in Sect. 2, the EFW instrument cannot obtain the full electric field vector. However, we can easily obtain an EFW electric field component which is almost parallel to the MAG $\beta$ direction. The green solid line in Fig. 4 corresponds to $E_{\beta} J_{\beta}$ calculated from the EFW $\beta$ component of the electric field. However, only the EFW electric fields from S/C 1, 3, and 4 are included in the average $E_{\beta}$, to make the averaging procedure the same for EFW and CIS/CODIF.

From Fig. 4 we see that CODIF and EFW agree well on the general trends (the green and solid red curves closely follow each other) in the power density and they both show sharp negative gradients for all CGRs. The HIA instrument shows a similar behaviour, although it does not observe CGR2. Comparing the corresponding thin curves and dashed lines, we note that most instruments also agree on the $E_{\beta} J_{\beta}$ component, constituting the dominant negative contribution during these intervals. Hence, at least two out of three Cluster electric field instruments agree on the location of the four CGRs. Moreover, we also see that the curves agree on the slowly sloping trend during more or less the entire event.

Note that the MAG system only differs from CGR by a few degrees for this event. Hence, after having investigated the CGRs in Fig. 4 in detail in the MAG system, in the following we will abandon this strict separation between the MAG and GSE systems and instead only refer to GSE.

\subsection{FAST observations}

To establish that these CGRs are related to the aurora we use magnetically conjugate data from the FAST satellite. During our 3.5-h long event, there are two close conjunctions between Cluster and FAST. In Fig. 3 these conjunctions 


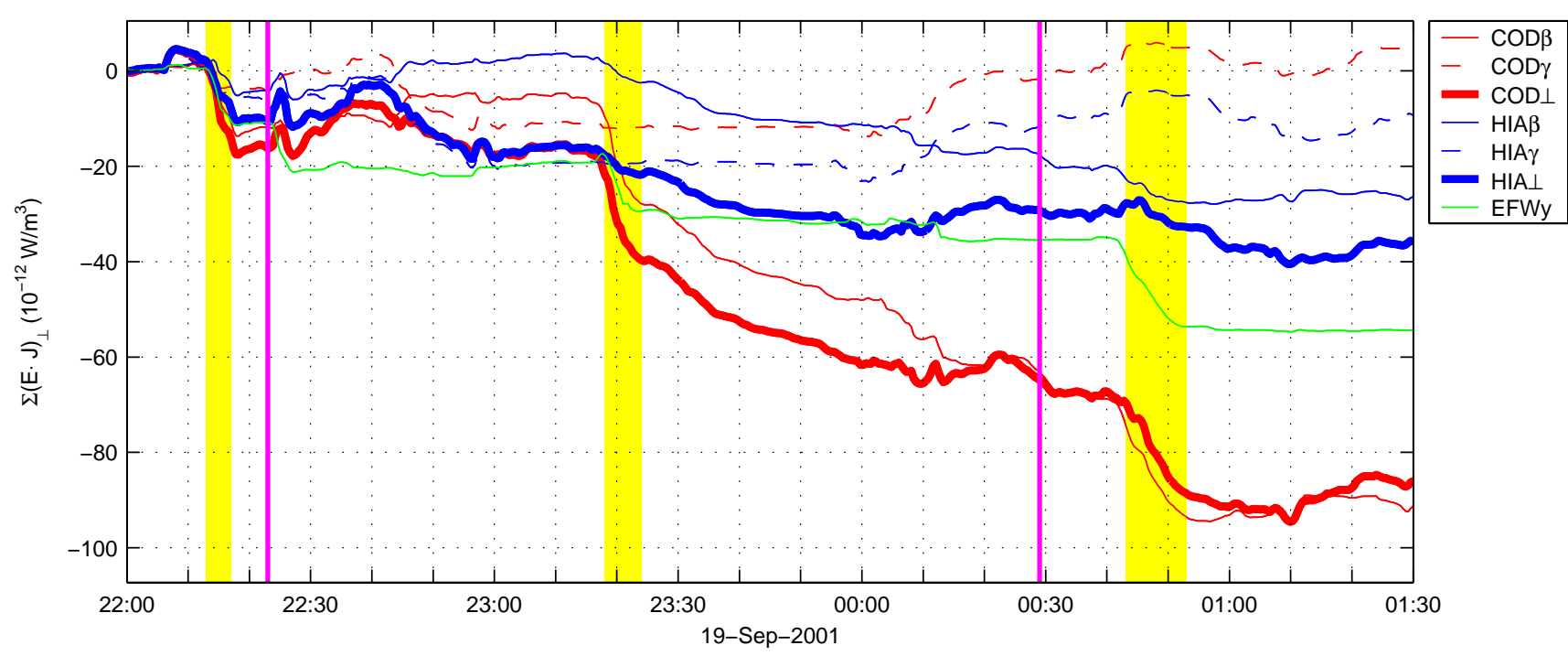

Fig. 4. Cumulative sums of the power density (thick lines) and the corresponding contributions from the perpendicular components in the MAG system, $E_{\beta} J_{\beta}$ (solid lines) and $E_{\beta} J_{\beta}$ (dashed lines). Data from the CODIF (red) and the HIA experiment (blue) are shown. The green line shows $E_{\beta} J_{\beta}$ calculated from one of the EFW electric field components which is very close to the MAG $\beta$ direction. The concentrated generator regions CGR1-CGR3 (indicated with yellow bands) appear when there are sharp gradients in the cumulative sums. The conjunctions with the FAST satellite are shown with the vertical magenta lines.

(a)

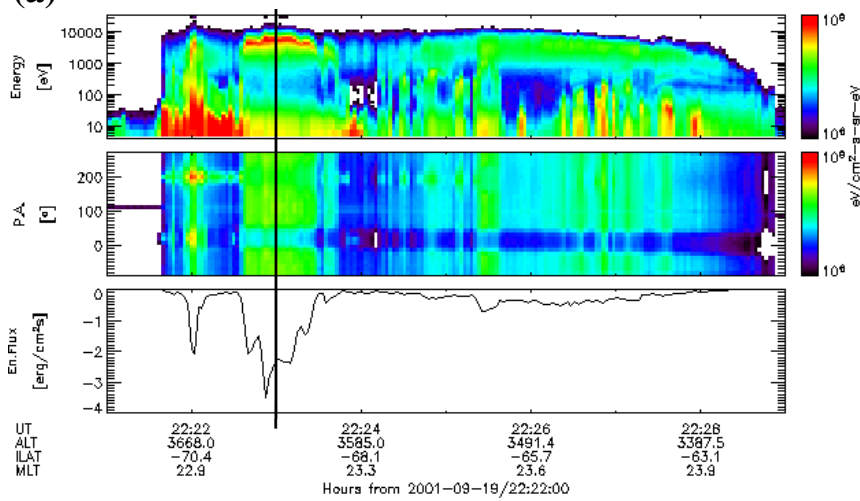

(b)

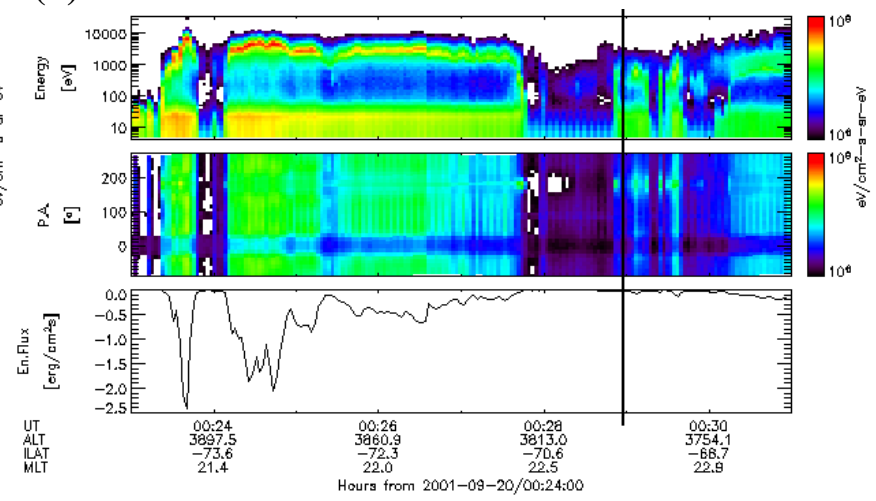

Fig. 5. FAST data for (a) the conjunction at 22:23 UT, on 19 September 2001, and (b) the conjunction at 00:29 UT, on 20 September 2001. The actual conjunctions are indicated by the vertical black lines. The top and middle panels show the electron energy and pitch angle spectrograms. The bottom panels show the electron energy flux at the satellite level.

are indicated with vertical magenta lines. According to the mapping the conjunctions occur at 22:23 UT (19 September 2001) and 00:29 UT (20 September 2001). Summary plots of the FAST data for the two conjunctions can be found in Figs. $5 \mathrm{a}$ and $\mathrm{b}$, where the conjunctions are marked with vertical black lines. The top and middle panels of Figs. 5a and $\mathrm{b}$ show electron energy and pitch-angle spectrograms, and the third panel the electron energy flux observed at the satellite level (to map to the ionosphere, this flux should be multiplied with a factor of four).
As discussed in Sect. 2 the low magnetospheric activity during our event reduces complications in the field line mapping due to magnetic field variations. Moreover, seeing similar signatures in the Cluster and FAST particle data also supports the mapping: In Fig. 3 we see that the magnetotail motion makes Cluster enter the plasma sheet at 22:13 UT while in Fig. 5a we see that FAST enters plasma sheet magnetic field lines at 22:21:30 UT. At the first conjunction, both Cluster and FAST are probing plasma sheet magnetic field lines just inside the boundary. 


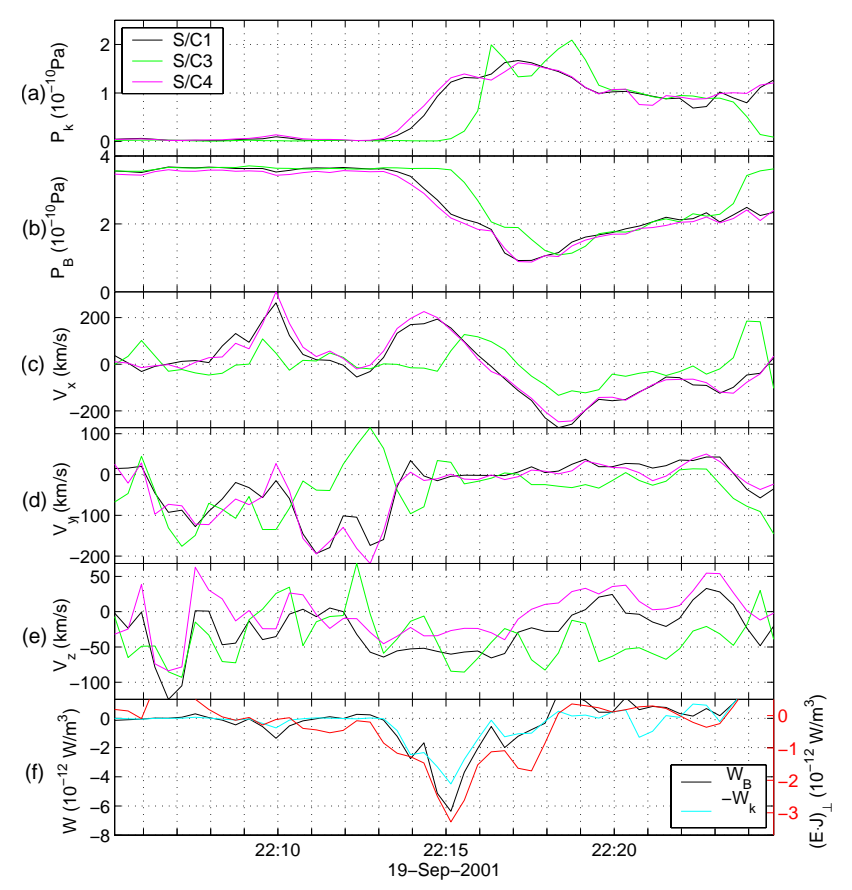

Fig. 6. Cluster data for CGR1. (a) Parallel ion pressure obtained by S/C 1, 3, and 4; (b) magnetic pressure at S/C 1, 3, and 4; (c-e) plasma flow velocities expressed in GSE $x, y$, and $z$ for S/C 1, 3, and 4; (f) power density (red) together with the rate at which work is done by the ion and magnetic pressure forces, $-W_{K}$ and $W_{B}$, respectively. Note the different scales of the power density, $-W_{K}$, and $W_{B}$ in panel (f).

As can be seen from the two top panels in Fig. 5, the FAST data show evidence of accelerated auroral electrons with a loss-cone distribution around the conjunction time. A more detailed investigation of the FAST electron distributions (not shown here) confirms these observations. Moreover, investigating the ion distributions (not shown here) we also find transversely heated oxygen ions. The electron and ion data, hence, indicate auroral activity around the first conjunction time.

Comparing with the Cluster data in Fig. 3 we see that the first conjunction is just after CGR1. However, as discussed above, we do not expect Cluster and FAST to be on exactly the same but only nearby field lines. Although the magnetic field line mapping may be somewhat uncertain due to the large distance between the spacecraft, we find that the agreement between the FAST and Cluster data supports the interpretation that CGR1 is correlated with auroral activity.

As shown in Fig. 3, the second conjunction at 00:29 UT occurs approximately $15 \mathrm{~min}$ before Cluster enters CGR3. Hence, it is difficult to draw any immediate conclusions about the auroral activities at lower altitudes exactly during CGR3. On the other hand, inspecting Fig. 5b we see that the conjunction occurs about one minute after FAST leaves an inverted- $\mathrm{V}$ region. This can be used to interpret the data. We use the information from Sect. 2 that the FAST and Cluster footprints in the ionosphere are close and that FAST and Cluster are moving approximately in opposite directions. Moreover, the magnetospheric activity is low $\left(K_{p}=1\right.$ during the entire event), which suggests rather stationary auroral structures. Hence, our data are consistent with FAST leaving a rather stationary auroral structure while it approaches the conjunction point from the south east. Cluster instead leaves the conjunction point behind as it approaches the plasma sheet boundary where CGR3 is located (the location of CGR3 close to the plasma sheet boundary is supported by the low value of the plasma beta, as shown in Fig. 3 in M06). Therefore, we conclude that the CGR3 observed by Cluster might well be correlated with auroral activity on neighboring magnetic field lines, even though the conjunction conditions are not exactly satisfied at the time of CGR3.

\section{The concentrated generator regions}

Figure 6 shows a more detailed picture of CGRs 1, which occur between 22:10 and 22:20 UT. When analyzing these data, it is important to know that relative to the center of the mass of the Cluster tetrahedron, the coordinates in kilometers along the GSE axes are $(700,-850,0)$ for S/C 1, (-50, 450, $-1100)$ for $S / C 3$, and $(-980,-500,280)$ for $S / C 4$. The top panel (a) shows the parallel ion pressure determined from the CODIF measurements on spacecraft 1,3 , and 4 . The pressure is mostly isotropic throughout the event (not shown). The transition from the lobe into the plasma sheet is clearly seen between 22:13 UT and 22:16 UT. Note that the pressure increase is first seen on S/C 1 and 4, which are closer to the equatorial plane. The magnetic pressure $P_{B}=B^{2} /\left(2 \mu_{0}\right)$, shown in panel (b), decreases according to the order the spacecraft enter the plasma sheet. The plasma flow velocities are shown in panels (c-e). Investigating the dynamic pressure caused by the plasma flow (not shown here) and using the density measured by CODIF, it is found that the plasma deceleration cannot provide enough power to explain the generation of electromagnetic energy.

To investigate the energy conversion within the CGRs in more detail, ultimately one must consider the full Poynting theorem. However, before we address this theorem we make a rough estimate of the work done by the plasma and magnetic pressure forces. Investigating CGR1 in more detail we first note that $W \sim-\boldsymbol{V} \cdot \nabla P$ can be interpreted as the rate (per unit volume) at which work is done by the pressure forces. With only three satellites we can unfortunately not estimate all components of the pressure gradients. From the spacecraft coordinates given above we see that reasonable estimates of the derivatives in the $x$ direction can be obtained by combining S/C 1 and S/C 4, and by including $\mathrm{S} / \mathrm{C} 3$ we can calculate derivatives in a direction approximately $45^{\circ}$ from both the $y$ and $z$ axes. If we use these derivatives, denoted $\partial_{\perp}$, as rough estimates of $\partial_{y} \sim-\partial_{\perp}$ and 


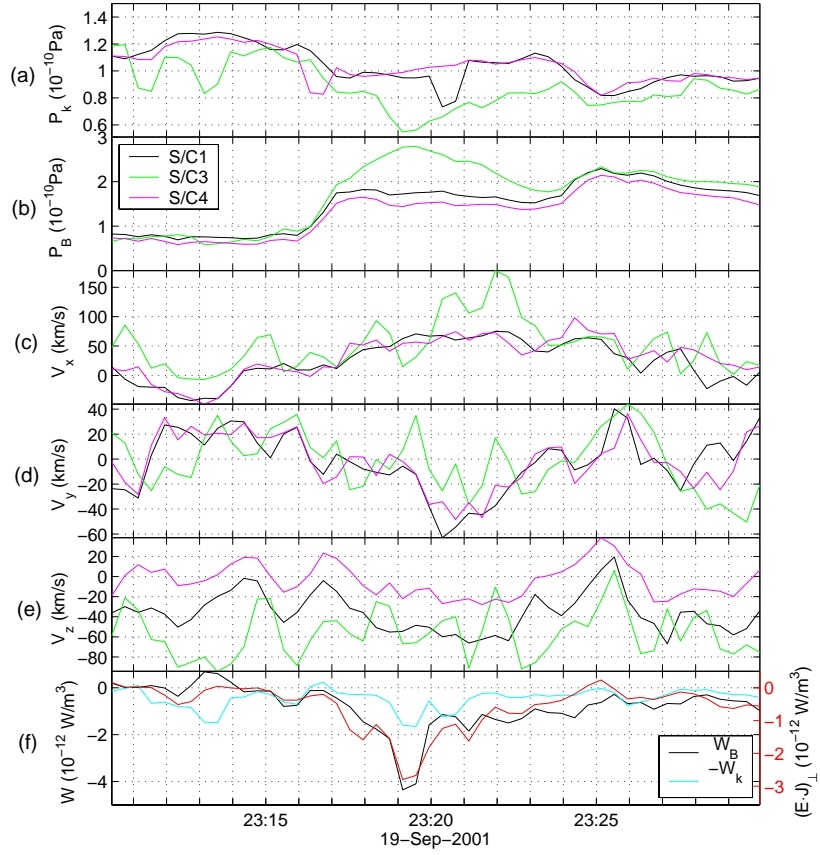

Fig. 7. The same as for Fig. 6 but for CGR2.

$\partial_{z} \sim \partial_{\perp}$, we can relate the curves in panel (f) to the rate at which work is done by the plasma and magnetic pressure (a more detailed calculation confirms $\partial_{y} \sim-\partial_{z}$ and implies that the plasma sheet boundary is tilted $45^{\circ}$ in the $y z$-plane). In Fig. 6f we have plotted $-W_{K}=V_{x} \partial_{x} P_{x}+\left(V_{z}-V_{y}\right) \partial_{\perp} P_{\perp}$ and $W_{B}=-V_{x} \partial_{x} P_{B}-\left(V_{z}-V_{y}\right) \partial_{\perp} P_{B}$, where $P_{x}$ and $P_{\perp}$ are the almost equal parallel and perpendicular plasma pressures. In the calculations of $-W_{K}$ and $+W_{B}$ we have used $V_{x}, V_{y}$, and $V_{z}$ as averages over the velocities observed by the three spacecraft.

CGR1 is defined by the negative values of $\boldsymbol{E}_{\perp} \cdot \boldsymbol{J}_{\perp}$, seen as a valley in the red curve in panel (f) between 22:13 UT and 22:18 UT. From the other curves in panel (f) we see that $-W_{K}$ and $W_{B}$ are also negative during most of this time interval, which supports the conclusion that the plasma and magnetic pressures push in opposite directions. We see that $W_{K}$ is positive and $W_{B}$ is negative. Hence, we conclude that the plasma pressure is doing work against the magnetic pressure.

To investigate CGR1 in more detail we address the Poynting theorem

$\partial_{t} B^{2} /\left(2 \mu_{0}\right)+\nabla \cdot \boldsymbol{S}=-\boldsymbol{J} \cdot \boldsymbol{E}$,

where $S$ is the Poynting flux and $B^{2} /\left(2 \mu_{0}\right)=P_{B}$ is the magnetic energy density or pressure. The electric field energy density is neglected since it is very small during our event. First estimating $\partial_{t} B^{2} /\left(2 \mu_{0}\right)$ from the Cluster data, we note that the satellites are practically stationary with respect to the plasma flow (the spacecraft velocity is about $1 \mathrm{~km} / \mathrm{s}$ while the plasma velocity is $\gtrsim 50 \mathrm{~km} / \mathrm{s}$ during CGR 1 ). Hence, the

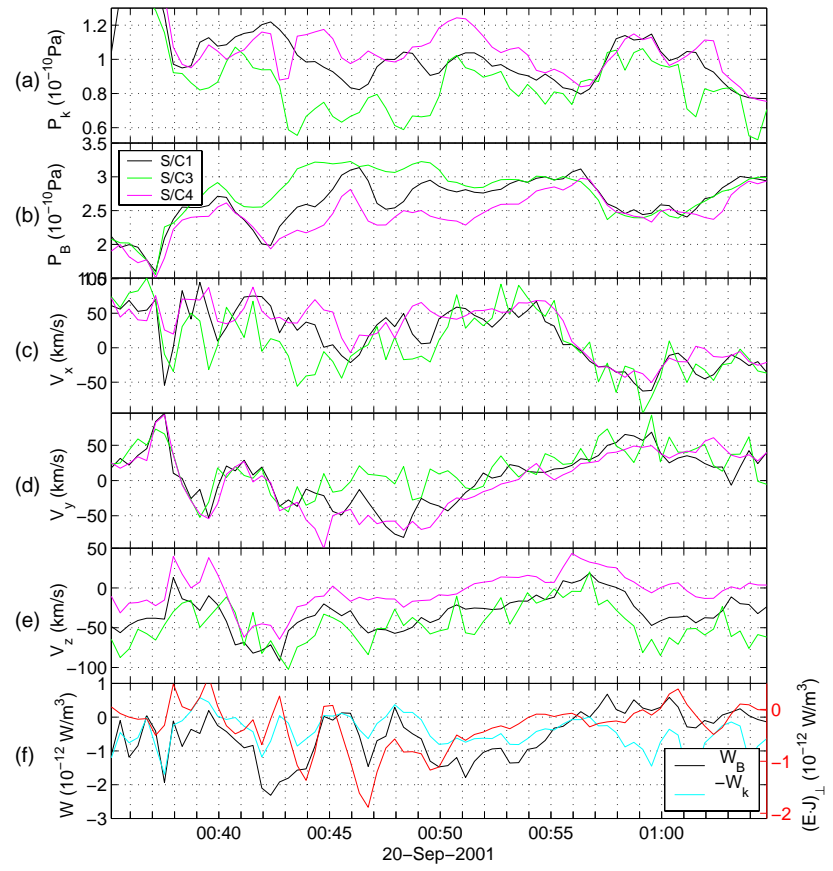

Fig. 8. The same as for Fig. 6 but for CGR3.

partial derivative $\partial_{t} B^{2} /\left(2 \mu_{0}\right)$ can be obtained by estimating the slope of the time series in Fig. 6b. Between 22:14 and 22:17 there is a decrease in the magnetic pressure (energy density) of about $0.2 \mathrm{nPa}$ first seen on $\mathrm{S} / \mathrm{C} 1$ and 4 , and thereafter on S/C 3. Therefore, $\partial_{t} B^{2} /\left(2 \mu_{0}\right) \approx-10^{-12} \mathrm{~W} / \mathrm{m}^{2}$, which is of the same sign and order as $\boldsymbol{E} \cdot \boldsymbol{J}$ during the time interval. Therefore $\nabla \cdot S$ must be positive for Eq. (1) to be satisfied and this is in accordance with a Poynting flux out from CGR1 and is also consistent with CGR1 being interpreted as a generator. Hence, we can conclude that electromagnetic energy is generated from the mechanical energy in the plasma pressure, and that some of the electromagnetic energy is transported away from CGR1 as a Poynting flux.

From the various curves in Fig. 6 we can construct a possible scenario for the evolution of CGR1. This is illustrated by the diagram in Fig. 9, where we show a projection on the GSE $(x, z)$ plane. Early in the event, at 22:14 UT, the ion pressure is starting to increase, first at S/C 4 and somewhat later at $\mathrm{S} / \mathrm{C} 1$, but not yet at $\mathrm{S} / \mathrm{C} 3$. The plasma flows at S/C 1 and S/C 4 are mainly earthward $\left(V_{x} \sim 200 \mathrm{~km} / \mathrm{s}\right)$, with a small southward component $\left(V_{z} \sim-50 \mathrm{~km} / \mathrm{s}\right)$. A similar southward flow is seen on $\mathrm{S} / \mathrm{C} 3$, but $V_{x}$ is insignificant. The magnetic field is slightly reduced at S/C 1 and S/C 4, but at S/C 3 the magnetic field is still unperturbed. This situation is sketched in the top panel of Fig. 9, where the shading indicates the ion pressure and the black arrows represent the observed plasma flows. The white arrow indicates a suggested plasma inflow and the boundary between the plasma sheet and the lobe is indicated with the thick black curve. 

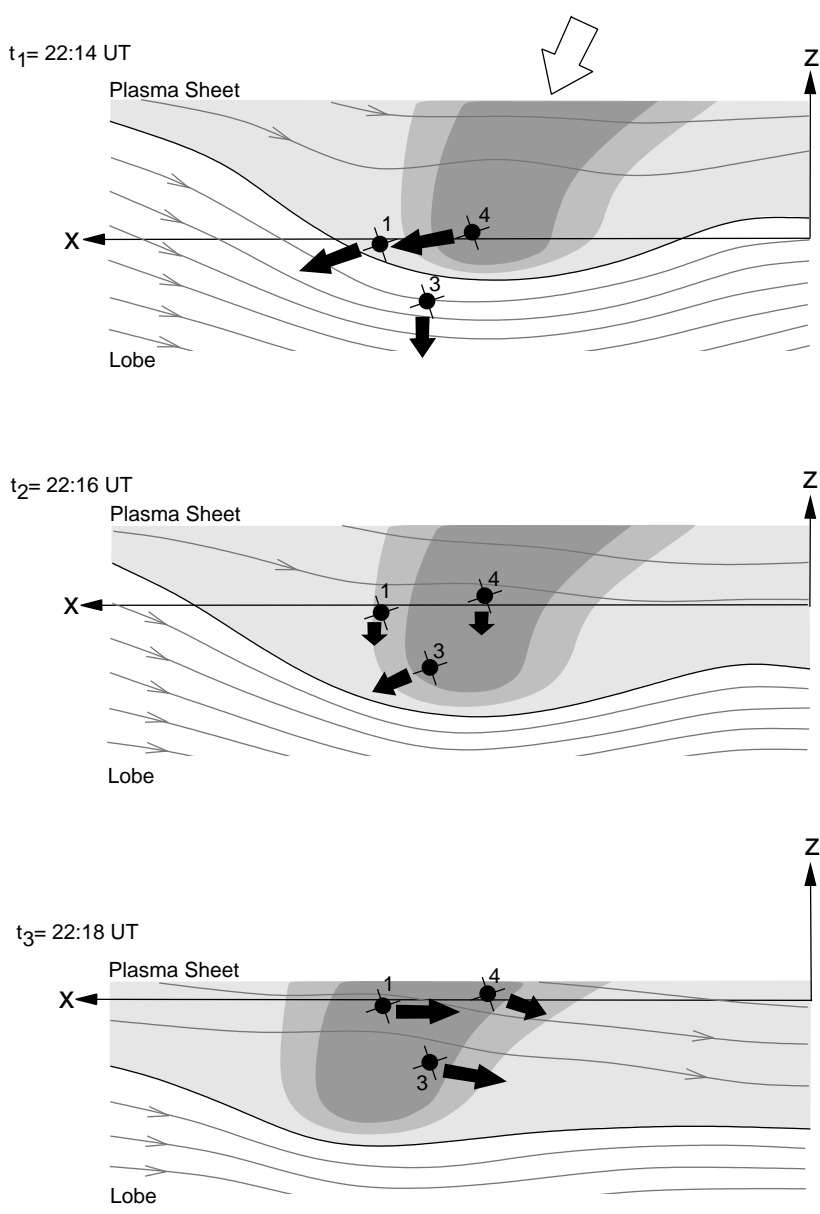

Fig. 9. A possible scenario for the evolution of CGR1. See the text for an explanation.

Two minutes later, at 22:16 UT, the pressure differences between the spacecraft are significantly reduced, mainly because of the strong pressure increase at S/C 3. There is still a rather uniform southward flow of the order $50 \mathrm{~km} / \mathrm{s}$, but the earthward flow at S/C 1 and S/C 4 has stopped. Instead, we now see an earthward flow of $100 \mathrm{~km} / \mathrm{s}$ at $\mathrm{S} / \mathrm{C} 3$.

At 22:18 UT, CGR1 has essentially passed over the satellites, as indicated in the bottom panel of Fig. 9. At this time, no significant spatial gradients are seen in the ion pressure or in the magnetic pressure. The plasma flows are mainly tailward, but at S/C 3 there is still also a southward flow.

We believe that the perturbation travelling over the spacecraft is associated with a plasma sheet thickening, as is expected to occur during the recovery phase of a substorm. This is indicated in Fig. 9. It is worth mentioning that the thickening of the plasma sheet might be associated with an irreversible energy change, as one would expect, related to the generator and the auroral activity. However, we cannot exclude the possibility of only a passing plasma pulse wave.

The normal, large-scale convection pattern in the Southern Hemisphere is toward the equatorial plane (with $V_{z}$ positive).
The small-scale CGR1 behaves differently. We see a region of enhanced plasma pressure that causes a southward expansion of the plasma sheet, propagating earthward.

Since $V_{y}$ remains small, CGR1 can reasonably well be described by a 2-D picture. Unfortunately, CGRs 2 and 3 are not equally simple. In Figs. 7 and 8 we see that all velocity components vary substantially during CGR 2 and CGR3. This indicates that these CGRs are essentially 3-D, and thus difficult to analyze in detail without more information about the gradients in the $(y, z)$ plane. However, the correlation between $\boldsymbol{E}_{\perp} \cdot \boldsymbol{J}_{\perp},-W_{K}$, and $W_{B}$ seen also during these events supports our interpretation that they are concentrated generator regions.

From the previous section we find that the main contribution to the power density comes from $E_{y} J_{y}$. This is true for all CGRs except for CGR1, where both the $y$ and $z$ components contribute approximately equally. For CGR1 we see clear drops in both the CODIF $y$ and $z$ components, in the HIA $z$ component and the related EFW $y$ component. However, the $z$ components are dominant during the first part of CGR1, while the $y$ components dominate the second part.

For the $y$ contribution the current and electric field behave similarly for CGR1-3: the current is large and positive while the corresponding electric field is slightly negative (the electric field is in fact slightly less than zero during most of our event on 19-20 September 2001). However, for the $z$ contribution to CGR1 the roles of the current and the electric fields are interchanged. Here the electric field shows a large positive peak in the $z$ direction while the corresponding current is negative.

We conclude that CGRs $2-3$ are activated by a strong increase in the current in the $y$ direction. Hence, the CGRs appear when there is a strong current toward dusk, while the corresponding electric field component is weak but directed towards dawn. We believe that the strong current is observed when Cluster is close enough to the boundary of the plasma sheet, so that it sees a pressure gradient and the associated diamagnetic current (see also M06, where such a scenario is discussed in more detail).

It should also be noted that CGR1 appears as Cluster enters the plasma sheet. Looking at the plasma flow velocity in Fig. 3b, we see that the CGR occurs in regions with considerable shears in the plasma flow in the GSE $x$ direction. During CGR1 there is a strong plasma flow in the sunward direction. During the next few minutes, between 22:17 and 22:24 UT, there is a significant flow in the tailward direction, and later on there is again a strong flow toward the Sun.

\section{Discussion}

Using conjugate FAST and Cluster data, in this article we have identified three concentrated generator regions (CGRs) in the plasma sheet. Cluster data from 22:00 UT, on 19 September, to 01:30 UT, on 20 September 2001, has 
been used. A fourth possible CGR around 02:30 UT, on 20 September 2001, has also been discussed in detail in M06. We note that all the CGRs are detected close to the plasma sheet boundary, as seen in the plasma beta (see Fig. $3 \mathrm{c}$ in M06).

As discussed in the previous section, the sign of the power density $\boldsymbol{E} \cdot \boldsymbol{J}$ can be established in certain regions by carefully comparing the different estimates based on the electric field inferred from the CIS/CODIF, the CIS/HIA, and measured by the EFW instruments, respectively. Investigating the three concentrated generator regions (CGRs) indicated in Fig. 4, we see that CODIF, HIA, and EFW agree well on the sign of the power density. However, it should be noted that the $\boldsymbol{E} \cdot \boldsymbol{J}$ in Fig. 4 is based on the electric field averaged over S/C 1, 3, and 4 for CODIF and EFW and over S/C 1 and 3 for HIA.

Moreover, from Fig. 4 we see that all instruments (CODIF, HIA, and EFW) also agree on a more slowly sloping trend in the power density during the first three hours of our event. Excluding the importance of random fluctuations and instrumental errors to this observation is out of the scope of this article. However, the fact that all instruments agree on this trend makes it plausible that the observed CGRs may be embedded within a larger region characterized by $\boldsymbol{E} \cdot \boldsymbol{J}<0$.

In this article we have observed that the main contribution to $\boldsymbol{E} \cdot \boldsymbol{J}<0$ comes from $E_{y} J_{y}$. As can be seen in Fig. 4, this trend is true for all the CGRs where the electric field, $E_{y}$, is small and negative. During CGR1 the plasma flow in the $y$ direction is almost zero. This is consistent with a local southward expansion of the plasma sheet passing over the satellites. For the other CGRs we see a non-negligible plasma flowing in the $y$ direction which suggests the passing of a more complicated, 3-D structure.

As discussed by Slavin et al. (2003) a small substorm took place around 20:39 UT and preceded the event investigated in this article. Intensifications were observed around 21:09, $21: 15$ and $21: 51$ and the recovery phase was initiated about 22:15. During this substorm Slavin et al. (2003) observed six travelling compression regions (TCRs) caused by bulges in the north-south thickness of the plasma sheet and which propagated with an average speed of about $400 \mathrm{~km} / \mathrm{s}$. All but one of the observed TCRs were moving toward the Earth. They are expected to form frequently as a result of simultaneous reconnection at a series of $\mathrm{X}$-lines.

The CGRs discussed in this article can also be interpreted as some complicated structures passing over the satellites, but the relation between CGRs and TCRs is not clear. The CGRs typically last 5-10 min and are observed within the plasma sheet, while the TCRs last less than a minute and are seen outside the plasma sheet during the active phase of the substorm. One possibility is that the CGRs (especially CGR1 and CGR2) are the aftermath of the reconnection events believed to generate the TCRs (Slavin et al., 2003). Another possibility is that the CGRs are related to some dynamic or wavy structures of the plasma sheet boundary, as discussed in M06.
The energy transport from generators in the magnetosphere to the auroral acceleration region is given by the Poynting flux. In Fig. $3 \mathrm{~h}$ the field-aligned Poynting flux averaged over Cluster S/C 1, 3, and 4 is plotted. We see that the Poynting flux is earthward (negative) for CGR1 and 3. It should be noted that the magnitude of the Poynting flux depends on the background magnetic field model. However, using different interpolation schemes for the background field, the direction of the field-aligned Poynting flux never changes, and during CGR 1 and 3 the magnitude of the downgoing Poynting flux is $1-10 \mu \mathrm{W} / \mathrm{m}^{2}$.

In a simple field line mapping, which uses the ratio of the Cluster and FAST magnetic fields $13 \mu \mathrm{T} / 30 \mathrm{nT} \approx 400$ as an area mapping factor, we find that a typical value of the parallel Poynting flux, $5 \mu \mathrm{W} / \mathrm{m}^{2}$, maps to an energy flux of about $2 \mathrm{~mW} / \mathrm{m}^{2}$ or $2 \mathrm{erg} / \mathrm{cm}^{-2} \mathrm{~s}^{-1}$ at the FAST altitude. Comparing this with the electron energy flux obtained by FAST and plotted in the 3rd panels of Figs. 5a and b, we see that the mapped Poynting flux is approximately of the same order as the energy flux observed by FAST around the two conjunction.

It should be noted that the Poynting flux during CGR2 is positive and hence directed opposite as compared to the other CGRs. One possible explanation for this discrepancy can be found in the magnetic field data in Fig. 5d. Here we see that the GSE $x$ component of the magnetic field turns positive, i.e. earthward, during a short time period around 23:00 UT. Hence, Cluster makes a short excursion into the Northern Hemisphere around that time. Furthermore, in Fig. 5h we see that the Poynting flux is positive during an extended interval of time of approximately $30 \mathrm{~min}$ after 23:00 UT. We believe that CGRs1-3 are included in a larger region consisting of several CGRs scattered over and moving in the vicinity of the plasma sheet boundary. The parallel Poynting flux can be used to conclude which part of this region Cluster is probing. CGR1 and 3, where the Poynting flux is earthward, probably correspond to the more earthward part of this extended region. CGR2, on the other hand, where the Poynting flux is oppositely directed, is probably in the more tailward part of this region. Our observations of generator regions close to the plasma sheet boundary and a Poynting flux toward the Earth are consistent with Wygant et al. (2002) who observed Alfvénic activity and a net earthward field-aligned Poynting flux large enough to explain the generation of strong auroras.

From our investigations we cannot conclude for sure that all the Poynting flux observed by Cluster is generated within CGR1-3. Moreover, it may well be that the energy generated in the CGRs is not transported all the way down to the ionosphere but circulated locally. However, the good agreement between the Poynting flux observed by FAST and the electron energy flux obtained by FAST suggests that there is a net production of energy near the plasma sheet boundary and at least a part of this energy is transported to the auroral ionosphere near the polar cap boundary. 
Continuing the comparison with the FAST data, we try to estimate the approximate size of the CGRs. From Fig. $6 \mathrm{~b}$ we see that at 22:15 UT the difference in the magnetic pressure, $P_{B}$, between $\mathrm{S} / \mathrm{C} 1$ (or $\mathrm{S} / \mathrm{C} 4$ ) and $\mathrm{S} / \mathrm{C} 3$ briefly reaches about half of its total change during CGR1. Since the distance between S/C 1 and S/C 3 in the $z$ direction is about $1100 \mathrm{~km}$, this suggests that the width of CGR 1 is about $2000 \mathrm{~km}$ in this direction. Using a distance mapping factor in the $z$ (northsouth) direction of about 25 (M06), this corresponds to a scale length of about $80 \mathrm{~km}$ at FAST. Since FAST moves with a velocity of about $6 \mathrm{~km} / \mathrm{s}$ this corresponds to $13 \mathrm{~s}$ of FAST data which is approximately the size of the small-scale structures within the inverted-V in Fig. 5a. Similarly, the scale size for CGR3 is roughly consistent with the FAST data. This supports our interpretation that the high altitude Cluster observations are correlated with the auroral activity observed by FAST.

\section{Summary and conclusions}

We use conjugate data from the Cluster and FAST satellites to investigate plausible generator regions in the nightside auroral magnetosphere. From 22:00 UT, on 19 September, to 01:30 UT, on 20 September 2001, Cluster is in the Southern Hemisphere, close to apogee, where it probes the plasma sheet and lobe at an altitude of about 18 Earth radii. During this time there are two close magnetic conjunctions with the FAST satellite. The magnetic activity is low throughout our event, suggesting a rather stationary situation. This facilitates the magnetic field line mapping and the interpretation of the observations.

In this study we identify three concentrated generator regions (CGRs) near the boundary of the plasma sheet by searching for signatures of negative power densities, $\boldsymbol{E} \cdot \boldsymbol{J}<0$, in the Cluster data. From the conjugate FAST data we see that the CGRs correlate with auroral precipitation. The downward Poynting flux observed by Cluster, as well as the scale size of the CGRs, are both consistent with the electron energy flux and the small-scale structures of the inverted-V regions observed by FAST.

The obtained power densities, $\boldsymbol{E} \cdot \boldsymbol{J} \sim 10^{-12} \mathrm{~W} / \mathrm{m}^{3}$, are consistent with a rough order of magnitude estimate of what is needed to power auroras. These power densities result from electric fields and current densities close to the measuring limits of the Cluster instruments. Obtaining accurate estimates of the power density is hence rather complicated, but the sign and the general trends are easier to obtain.

Apart from the four observed CGRs, we also see some indications of more extended regions of weakly negative values of the power density. However, to be able to determine whether this signature correspond to extended generator regions or are just artefacts due to instrumental errors or random fluctuations, a more extensive investigation is needed.
The CGRs discussed in this article are observed near the boundary of the plasma sheet where there are strong gradients in the plasma pressure. The associated diamagnetic current in the positive GSE $y$ direction, together with a negative $E_{y}$, cause the main contribution to the power density within these CGRs. Our observations are consistent with a local southward expansion of the plasma sheet moving over the Cluster satellites (CGR1) and/or rather the passing of complicated, 3-D wavy structures. We conclude that there is a net production of energy near the plasma sheet boundary and that this energy is transported to the auroral ionosphere near the polar cap boundary.

We find that the ion pressure is doing work against the magnetic pressure, and that kinetic energy is being converted to electromagnetic energy. We estimate the divergence of the Poynting flux and conclude that some of the energy is transported away from the CGR1. The remaining CGR2 and CGR3 are much more difficult to analyze due to their pronounced 3-D character. However, the correlation between the rate at which work is done by the ion and magnetic pressure forces supports our interpretation that they are concentrated generator regions.

To our knowledge, these are the first in-situ observations of the crossing of an auroral generator region. We believe that the observed CGRs are related to auroral activity and to an irreversible energy change.

Acknowledgements. We are pleased to thank A. Blăgău, M. Bouhram, T. Karlsson, Y. Khotyaintsev, G. Marklund, G. Paschmann, and H. Vaith for fruitful discussions. O. Marghitu acknowledges the kind hospitality of Max-Planck-Institut für extraterrestrische Physik, Garching, and the support by the German Bundesministerium für Bildung und Forschung and the Zentrum für Luft- und Raumfahrt under contract 50 OC 0102. The work in Romania was funded through the program AEROSPAŢIAL, contracts 72/2003 PROSPERO and 118/2004 DIAFAN. We also acknowledge the World Data Center for Geomagnetism, Kyoto, for the $D_{s t}$ index, the GeoForschungsZentrum (GFZ), Potsdam, for the $K_{p}$ index, the ACE magnetic field instrument (MFI), and plasma instrument (SWEPAM) teams, for the solar wind parameters, as well as the Satellite Situation Center (SSCWeb), for the conjunction query tool.

Topical Editor T. Pulkkinen thanks J. Vogt and another referee for their help in evaluating this paper.

\section{References}

Angelopoulos, V., Baumjohann, W., Kennel, C. F., Coroniti, F. V., Kivelson, M. G., Pellat, R., Walker, R. J., Lühr, H., and Paschmann, G.: Bursty bulk flows in the inner central plasma sheet, J. Geophys. Res., 97, 4027-4039, 1992.

Balogh, A., Carr, C. M., Acuña, M. H., et al.: The Cluster Magnetic Field Investigation: overview of in-flight performance and initial results, Ann. Geophys., 19, 1207-1217, 2001.

Birn, J., Hesse, M., Haerendel, G., Baumjohann, W., and Shiokawa, K.: Flow braking and the substorm current wedge, J. Geophys. Res., 104, 19895-19903, 1999. 
Borovsky, J. E.: Auroral arc thicknesses as predicted by various theories, J. Geophys. Res., 98, 6101-6138, 1993.

Boström, R.: Mechanisms for driving Birkeland currents, in Physics of the Hot Plasma in the Magnetosphere, edited by: Hultqvist, B. and Stenflo, L., Plenum Press, New York, 341-362, 1975.

Carlson, C., McFadden, J., Turin, P., Curtis, D., and Magoncelli, A.: The electron and ion plasma experiment for FAST, Space Sci. Rev., 98, 33-66, 2001.

Dunlop, M. W., Balogh, A., Glassmeier, K.-H., and Robert, P.: Four-point Cluster application of magnetic field analysis tools: The Curlometer, J. Geophys. Res., 107, doi:10.1029/2001JA005089, 2002.

Escoubet, C. P., Schmidt, R., and Goldstein, M. L.: Cluster - Science and mission overview, Space Sci. Rev., 79, 11-32, 1997.

Escoubet, C. P., Fehringer, M., and Goldstein, M. L.: The Cluster mission - Introduction, Ann. Geophys., 19, 1197-1200, 2001.

Gustafsson, G., Bostrom, R., Holback, B., Holmgren, G., Lundgren, A., Stasiewicz, K., Ahlen, L., Mozer, F. S., Pankow, D., Harvey, P., Berg, P., Ulrich, R., Pedersen, A., Schmidt, R., Butler, A., Fransen, A. W. C., Klinge, D., Thomsen, M., Falthammar, C. G., Lindqvist, P. A., Christenson, S., Holtet, J., Lybekk, B., Sten, T. A., Tanskanen, P., Lappalainen, K., and Wygant, J.: The electric field and wave experiment for the Cluster mission, Space Sci. Rev., 79, 137-156, 1997.

Gustafsson, G., André, M., Carozzi, M. T., et al.: First results of electric field and density observations by Cluster EFW based on initial months of operation, Ann. Geophys., 19, 1219-1240, 2001.

Janhunen, P. and Olsson, A.: New model for auroral acceleration: O-shaped potential structure cooperating with waves, Ann.Geophys., 18, 596-607, 2000.

Lu, G., Brittnacher, M., Parks, G., and Lummerzheim, D.: On the magnetospheric source regions of substorm-related field-aligned currents and auroral precipitation, J. Geophys. Res., 105, 18483 $18493,2000$.
Lysak, R. L.: Electrodynamic coupling of the magnetosphere and ionosphere, Space Sci. Rev., 52, 33-87, 1990.

Marghitu, O., Hamrin, M., Klecker, B., et al: Experimental investigation of auroral generator regions with conjugate Cluster and FAST data, Ann. Geophys., 24, 619-635, 2006.

Paschmann, G. and Daly, P. W. (Eds.): Analysis methods for multispacecraft data, ISSI, ESA, Bern, 1998.

Paschmann, G., Quinn, J. M., Torbert, R. B., et al.: The electron drift instrument on Cluster: overview of first results, Ann. Geophys., 19, 1273-1288, 2001.

Paschmann, G., Haaland, S., and Treumann, R.: Auroral plasma physics, vol. 103, Kluwer Academic Publishers, Dordrecht, 320324, 2003.

Rème, H., Aoustin, C., Bosqued, J. M., et al.: First multispacecraft ion measurements in and near the Earth's magnetosphere with the identical Cluster ion spectrometry (CIS) experiment, Ann. Geophys., 19, 1303-1354, 2001.

Rostoker, G. and Boström, R.: A mechanism for driving the gross Birkeland current configuration in the auroral oval, J. Geophys. Res., 81, 235-244, 1976.

Slavin, J. A., Owen, C. J., Dunlop, M. W., Borälv, E., Moldwin, M. B., Sibeck, D. G., Tanskanen, E., Goldstein, M. L., Fazakerley, A., Balogh, A., Lucek, E., Richter, I., Rème, H., and Bosqued, J. M.: Cluster four spacecraft measurements of small traveling compression regions in the near tail, Geophys. Res. Lett., 30, doi:10.1029/2003GL018438, 2003.

Vogt, J.: Alfvén wave coupling in the auroral current circuit, in Surveys in Geophysics, vol. 23, Kluwer Academic Press, 335377, 2002.

Wygant, J. R., Keiling, A., Cattell, C. A., Lysak, R. L., Temerin, M., Mozer, F., Kletzing, C. A., Scudder, J. D., Streltsov, V., Lotko, W., and Russell, C. T.: Evidence for kinetic Alfven waves and parallel electron energization at $4-6 R_{E}$ altitudes in the plasma sheet boundary layer, J. Geophys. Res., 107, doi:10.1029/2001JA900113, 2002. 